\title{
Aus der DGI
}

https://doi.org/10.1515/iwp-2019-2032

\section{DGI-Mitgliederversammlung 2019}

Die DGI-Mitgliederversammlung für das Geschäftsjahr 2018 wird im Rahmen des DGI-Forums Wittenberg am 18. September 2019, 14:00 Uhr, in der LEUCOREA, Collegienstraße 62, 06886 Lutherstadt Wittenberg stattfinden. Wir freuen uns, wenn viele DGI-Mitglieder teilnehmen.

\section{DGI-Fort- und}

\section{Weiterbildungsangebot im Herbst und Winter 2019}

Nach dem Sommerloch nimmt die DGI ihr Fort- und Weiterbildungsprogramm wieder auf. Themenschwerpunkte sind: professionelle Kommunikation, formale und inhaltliche Erschließung, digitale Teamarbeit, EU-DSVGO, Urheberrecht, Wissensmanagement sowie Projektplanung, -durchführung und -evaluierung. Einzelne Lehreinheiten werden in einem zweistufigen Seminaraufbau angeboten. So können je nach Wissensstand entweder das Basis-Modul, das Fortgeschrittenen-Seminar oder beide besucht werden.

- Kommunikation für Information Professionals I 28. Oktober 2019, 9:30-17:00, Frankfurt am Main: Modul 1 - Zielorientiert und erfolgreich kommunizieren, Theorie und praktische Übungen

- Kommunikation für Information Professionals II 29. Oktober 2019, 9:30-17:00, Frankfurt am Main: Modul 2 - Rhetorik und Präsentationstechniken: Ideen und Produkte überzeugend vermitteln

- Methoden und Instrumente der formalen und inhaltlichen Informationserschließung I 4. November 2019, 9:30-17:00, Frankfurt am Main: Modul 1 Metadaten gegen das Informationschaos - Einführung in die formale und inhaltliche Erschließung

- Methoden und Instrumente der formalen und inhaltlichen Informationserschließung II 5. November 2019, 9:30-17:00, Frankfurt am Main: Modul 2 - Klassifikationen, Thesauri, Taxonomien und Ontologien

- DGI-Praxisseminar: EU-DSGVO 6. November 2019 , 9:30-17:00, Frankfurt am Main: Vorgaben zur Auftragsdatenverarbeitung, Umgang mit Kundendaten, Dokumentationspflichten
- Teambildung und Zusammenarbeit im digitalen Zeitalter 14. November 2019, 9:30-17:00, Frankfurt am Main: Tools, Techniken, Erfolgsfaktoren

- Das Urheberrecht und seine Einhaltung 15. November 2019, 9:30-14:00, Frankfurt am Main: Urheberrecht in Unternehmen, Rechte der Autoren, internationale Bestimmungen, Dokumentlieferdienste, Lizenzen

- Wissensmanagement 2. Dezember 2019, 9:30-17:00, Frankfurt am Main: Wissenstransfer und Know-howSicherung in Unternehmen. Eine Einführung für Information Professionals

- Projektplanung, -durchführung und -evaluierung 9. Dezember 2019, 9:30-17:00, Frankfurt am Main: Einführung für Information Professionals

\section{Wir brauchen Ihre Meinung - DGI-Online-Umfrage 2019}

Im Jahr 2018 feierte die DGI ihren 70. Geburtstag. Für uns ein guter Anlass, Sie nach Ihren Wünschen, und Bedürfnissen, aber auch nach Kritik und Verbesserungsvorschlägen zu fragen: Wie stellen Sie sich die Arbeit der DGI zukünftig vor? Welche Ziele sollten wir uns setzen und was können wir über Bord werfen? Wir laden Sie herzlich ein, an unserer DGI-Online-Umfrage 2019 teilzunehmen. Diese ist vollständig anonym und in ca. 15 Minuten zu beantworten. Die Ergebnisse werden wir auf unserer Webseite und in dieser Zeitschrift veröffentlich. Zur Umfrage: https://www.umfra geonline.com/s/ed85eef\&id=d54ea09

Vielen Dank fürs Mitmachen!

\section{Lehrgang Informationsassistenz weiter erfolgreich}

Erleichtert und mit strahlenden Gesichtern haben die neu gekürten Informationsassistentinnen im Mai 2019 ihre DGI-Zertifikate entgegengenommen, die sie nach erfolgreicher Prüfung in den Räumen der DGI-Geschäftsstelle überreicht bekamen. In insgesamt vier Wochen zwischen dem 6. März und 17. Mai 2019 standen alle für unseren Berufsstand wichtigen Themen - von der Recherche in professionellen Datenbanken bei Genios, STN und WTI über die 
Aufbereitung von Rechercheergebnissen für Kunden bis zur inhaltlichen Erschließung von Medien aller Art, von Suchmaschinenoptimierung und Erarbeitung einer Social Media Strategie bis zu Thesauruskonstruktion, Urheberrecht und Lizenzierung - auf dem Lehrplan. Abwechslung brachten ein ganztägiger Workshop zur Wikipedia, ein Kommunikationsworkshop und die Führung bei der Deutschen Nationalbibliothek. Die Strukturierung des Lehrgangs in einzelne thematisch zusammenhängende Module ermöglichte es erneut, je nach individuellem Fortbildungsbedarf nur an einzelnen kompakten Lerneinheiten teilzunehmen und dafür eine qualifizierte Teilnahmebestätigung zu erhalten.

Der nächste Lehrgang soll im ersten Halbjahr 2020 stattfinden. Interessierte werden gebeten, sich möglichst frühzeitig telefonisch bei der DGI-Geschäftsstelle unter 069430313 oder per E-Mail unter informationsassistent@dgi-info.de zu melden, damit passende Termine abgestimmt werden können.

\section{Zusammenarbeit mit der ÖGDI}

DGI-Präsidentin Marlies Ockenfeld hielt am 28. Mai 2019 einen Impulsvortrag bei einer gemeinsamen Veranstaltung der Österreichischen Gesellschaft für Dokumentation und Information und der IG LektorInnen und WissensarbeiterInnen in der Universität Wien. Motto der dreistündigen Veranstaltung war Publish AND perish - wie publizieren, ohne sich zu verlieren. Am 29. Mai nahm sie an einer Vorstandssitzung der ÖGDI teil, bei der der Kooperationsvertrag zwischen DGI und ÖGDI im Mittelpunkt stand. Er sieht die gegenseitige Gewährung von Mitgliederrabatten, die Durchführung gemeinsamer Veranstaltungen und eine stärkere Nutzung der IWP für die Berichterstattung aus Österreich vor.

\section{Buchmesse 2019 mit DNI-Treffen}

Die DGI wird vom 16. bis 20. Oktober 2019 erneut mit dem b-i-t-verlag einen Gemeinschaftsstand in Halle 4.2 auf der Frankfurter Buchmesse haben.

Das Deutsche Netzwerk der Indexer (DNI) führt dort am Donnerstag, dem 17. Oktober, von 16:00 bis 17:30 Uhr sein traditionelles Mitgliedertreffen durch. Als Gast nimmt Heather Hedden aus den U.S.A. teil, die einen Vortrag über „Thesauri for indexing support“, d.h. wie die Registererstellung durch den Einsatz von Thesauri unterstützt werden kann, halten wird.
Heather Hedden ist Freelancer und bietet mit ihrem Business Hedden Information Management (www.hed den-information.com) den Aufbau und die Pflege von Thesauri und anderen kontrollierten Vokabularen sowie die Erstellung von Buchregistern an. Zudem gibt sie OnlineKurse und Workshops zur Erstellung von Thesauri und Vokabularen. In der Vergangenheit war sie auch als Thesaurus- und Vokabular-Entwicklerin bei verschiedenen Firmen angestellt: Gale/Cengage Learning, Project Performation Corporation, First Wind und Viziant Corporation. Sie gehörte dem Vorstand der American Society for Indexing (ASI) an und ist Autorin des Fachbuches The Accidental Taxonomist (Information Today-Verlag, 1. Aufl. 2010, 2. Aufl. 2016).

Vorstandsmitglieder der DGI werden am Stand für Gespräche zur Verfügung stehen und freuen sich auf den Austausch mit Mitgliedern und interessierten Gästen.

\section{Nicole Walger leitende Bibliotheksdirektorin in Duisburg}

Zum 1. Mai 2019 hat die Sprecherin der DGI-Fachgruppe Akademische Integrität, Nicole Walger, die Leitung der Universitätsbibliothek Duisburg übernommen. Nicole Walger (41) studierte zunächst Germanistik und Romanistik an der Goethe-Universität in Frankfurt am Main, um dann noch ein Studium der Bibliotheks- und Informationswissenschaften an der Fachhochschule Köln anzuschließen. Ab 2011 leitete sie eine Bereichsbibliothek an der UB Mainz und wurde 2016 stellvertretende Leiterin der UB Siegen. Dort baute sie Strukturen und Dienste zum wissenschaftlichen Publizieren und zum Forschungsdatenmanagement auf. Die DGI gratuliert und wünscht alles Gute für die neue Position.

\section{Neuer Vorstand des MAID}

Der Münchener Arbeitskreis für Information und Dokumentation hat auf seiner Mitgliederversammlung am 17. Mai 2019 den Vorstand neu gewählt. Vorsitzende bleibt Dorothea Vötter, wiedergewählt wurden auch Dr. Marita Meisenheimer und Dr. Werner Wasner. Neue Mitglieder des Vorstands sind Eva-Maria Brünnel von der Gesellschaft für Informationsmanagement und Dokumentation $\mathrm{mbH}$ in Würzburg und Jan Strack vom Bayerischen Rundfunk. 


\section{DGI-Mitglieder stellen sich vor}

\section{Gudrun Schmidt}

Diplom-Pädagogin und Wissenschaftliche Dokumentarin, freiberufliche Beraterin, Dozentin und Moderatorin

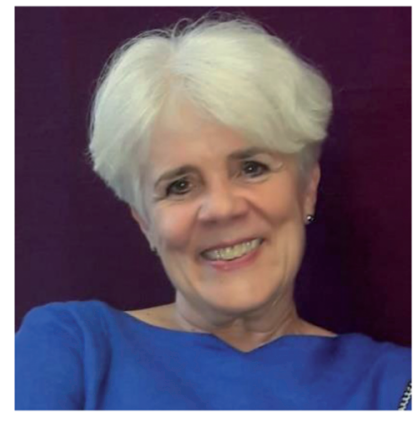

Meine Seminare und Workshops drehen sich um die Themen Information, Wissen und Kommunikation. Unter anderem führe ich regelmäßig verschiedene DGI-Praxisseminare durch. Ich bin seit vielen Jahren Mitglied der DGI und seit kurzem auch in der Gesellschaft für Wissensmanagement (GfWM) im Team der Regionalkoordinatoren Frankfurt/Rhein-Main aktiv. Einen Beitrag zur beruflichen und persönlichen Weiterbildung in unserer Branche zu leisten, liegt mir sehr am Herzen. Auch und gerade für uns Information Professionals ist es in Zeiten der Digitalisierung und immer schnellerer Umwälzungen in Gesellschaft und Arbeitsleben wichtiger als je zuvor, sich nicht nur fachlich, sondern auch im Hinblick auf fach- und branchenunabhängige Kompetenzen - wie Kommunikation, Eigenverantwortung, Teamfähigkeit, unternehmerisches Denken und Handeln - fit $\mathrm{zu}$ halten, uns stetig weiter $\mathrm{zu}$ entwickeln und zukunftssicher zu positionieren.

Schon während meiner Ausbildung Anfang der 1980er Jahre stellte sich mir die Frage, wie es am besten gelingen könnte, der Öffentlichkeit unseren Beruf und den Nutzen, den wir schaffen, zu vermitteln. Wenn jemand aus dem Kreis der Familie, Freunde, Nachbarn und Bekannten fragte „Was machst Du jetzt eigentlich beruflich?“ fiel es mir schwer, kurz, bündig und für meine Gesprächspartner befriedigend $\mathrm{zu}$ antworten. Der weitere Gesprächsverlauf ging in die Richtung: „So was ähnliches wie Bibliothekare, nur mit mehr Computer?“ Oder „Sowas wie Programmierer, nur irgendwie spezialisiert auf Fachliteratur?“ Den typischen Dokumentar, die typische Dokumentarin stellte sich meine Umgebung (und zugegeben, ich selbst auch, zumindest anfangs) als sehr gewissenhaft, gründlich, detailverliebt, dabei tendenziell eher introvertiert und nicht unbedingt sehr kommunikativ vor, das Berufsbild ins- gesamt wirkte auf Außenstehende eher tröge. Faktisch ist dieser „typische“ Vertreter unserer Berufsgruppe immer seltener anzutreffen, und das Berufsbild mutet differenzierter und moderner an. Dennoch stehen viele von uns immer noch oder gerade heute wieder, im Zeitalter von Internet, Digitalisierung und künstlicher Intelligenz, vor der Herausforderung, sich erklären und oftmals auch rechtfertigen zu müssen. Hier setzt meine Beratung und setzen meine Seminare und Workshops an.

Ich selbst kam eher zufällig, direkt nach dem Studium über einen ersten Job in der Bibliothek des DIPF mit unserer Branche in Berührung. Der Leiter der Bibliothek empfahl mir, den Jahreslehrgang für Wissenschaftliche Dokumentare am damaligen Lehrinstitut für Dokumentation in Frankfurt am Main zu absolvieren. Im Erststudium hatte ich Erziehungswissenschaften, Psychologie und Soziologie studiert und mit einem Diplom mit Schwerpunkt Erwachsenenbildung und außerschulische Jugendbildung, abgeschlossen. Dazu schien mir die Dokumentarausbildung eine sinnvolle Ergänzung zu sein, ging es doch auch hier, wie in meinem Studium, letztlich um Informationsaufbereitung und Wissenstransfer. Zudem schienen mir die Berufsaussichten rosig: In einer englischsprachigen Ausgabe der Cosmopolitan wurde damals in einem Ranking der karriereträchtigsten Berufe der Information Specialist bzw. Information Broker als Zukunftsberuf schlechthin aufgeführt, mit dem Versprechen: „They (Chefs, Kunden, Kollegen) will treat you like a honey bee“. Ganz so ist das zwar nicht eingetroffen, aber ich kann nicht klagen: Meine beiden Abschlüsse öffneten mir den Weg zu einem sehr spannenden, abwechslungsreichen und befriedigenden Berufsleben:

Da war zunächst die bibliothekarische und dokumentarische Arbeit im DIPF und anschließend beim deutschen Volkshochschulverband: Hier lernte ich von der Pike auf Katalogisierung, Inhaltserschließung und Thesaurusarbeit.

Es folgten Insgesamt über 20 Jahre in der Entwicklungszusammenarbeit, bei internationalen Organisationen und Projekten mit den Stationen: Konzeptionelle Arbeit und Leitung der zentralen Informations- und Dokumentationsstelle in der GTZ (heute GIZ), Aufbau einer Projektdokumentation, Koordination des Internetauftritts der GTZ, Mitarbeit im strategischen Unternehmensprojekt Wissensmanagement, Konzeption eines zentralen Wissensspeichers und Koordinationsaufgaben bei der Entwicklung neuer Geschäftsfelder, wie „IKT in der Entwicklungszusammenarbeit“. In diesem Kontext ergab sich auch die Möglichkeit, im Ausland zu leben und zu arbeiten: Ein Jahr als Visiting Expert für Wissensmanagement in der Welternährungsorganisation FAO in Rom, später je- 
weils rund zwei Jahre in Syrien und in Jordanien, mit Beratungseinsätzen in EU-Projekten in Syrien und Ägypten sowie an der Deutsch-Jordanischen Universität.

Nach Rückkehr aus dem Ausland folgten rund vier Jahre bei der WTI-Frankfurt eG, dem Fachinformationsvermittler für ingenieurwissenschaftliche Themen. Hier war ich als Leiterin des Vertriebs und der Geschäftsfeldentwicklung sowie im Vorstand tätig. Ich entwickelte ein Wissensspiel - TecGame -, das insbesondere für jüngere Zielgruppen einen etwas anderen Zugang zu den wissenschaftlich-technischen Datenbankinhalten eröffnete, und war an einem umfangreichen Thesaurus-Kooperationsprojekt gemeinsam mit der TIB beteiligt.

Zwischen diesen beruflichen Stationen ergaben sich immer wieder Lehr- und Beratungsaufträge, unter anderem von Hochschulen, dem Goethe-Institut sowie im Rahmen einer Workshop-Reihe im Tourismusbereich. Mit meiner aktuellen freiberuflichen Tätigkeit als Beraterin, Moderatorin und Dozentin knüpfe ich an diesen Erfahrungen an.
Ich freue mich sehr darüber, dass ich auch einige Praxisseminare bei der DGI anbieten und mich so mit Angehörigen meiner Berufsgruppe regelmäßig austauschen kann. Meine bisherigen Seminarthemen bei der DGI waren Kommunikation für Information Professionals sowie Metadaten/Thesauri. Weitere Seminare sind geplant. Die Kommunikationsseminare sind mein persönliches Highlight: Hier setzen wir uns theoretisch und in praktischen Übungen damit auseinander, wie wir unsere Informationsprodukte und -services mit Hilfe bewährter Methoden und Tools überzeugend erklären, und z.B. mit dem Elevator Pitch in wenigen Minuten auf den Punkt bringen können. Alle lernen mit- und voneinander, den Nutzen ihrer Arbeit herauszuarbeiten, und können im Idealfall dann bei der nächsten Gelegenheit auch auf die Frage „Was machen Sie denn eigentlich beruflich?“ präzise und überzeugend antworten.

Gudrun Schmidt gudrun.g.schmidt@gmail.com 